\title{
La conservation du mil par la potasse. Vers une épistémologie dogon des matières
}

\author{
Laurence Douny
}

\section{(2) OpenEdition}

Journals

Édition électronique

URL : https://journals.openedition.org/tc/8860

DOI : $10.4000 /$ tc. 8860

ISSN : 1952-420X

Éditeur

Éditions de l'EHESS

\section{Édition imprimée}

Date de publication : 17 mai 2018

Pagination : 34-37

ISBN : 978-2-7132-2750-9

ISSN : 0248-6016

\section{Référence électronique}

Laurence Douny, « La conservation du mil par la potasse. Vers une épistémologie dogon des matières », Techniques \& Culture [En ligne], 69 | 2018, mis en ligne le 17 mai 2020, consulté le 29 septembre 2022. URL : http://journals.openedition.org/tc/8860 ; DOI : https://doi.org/10.4000/tc.8860 


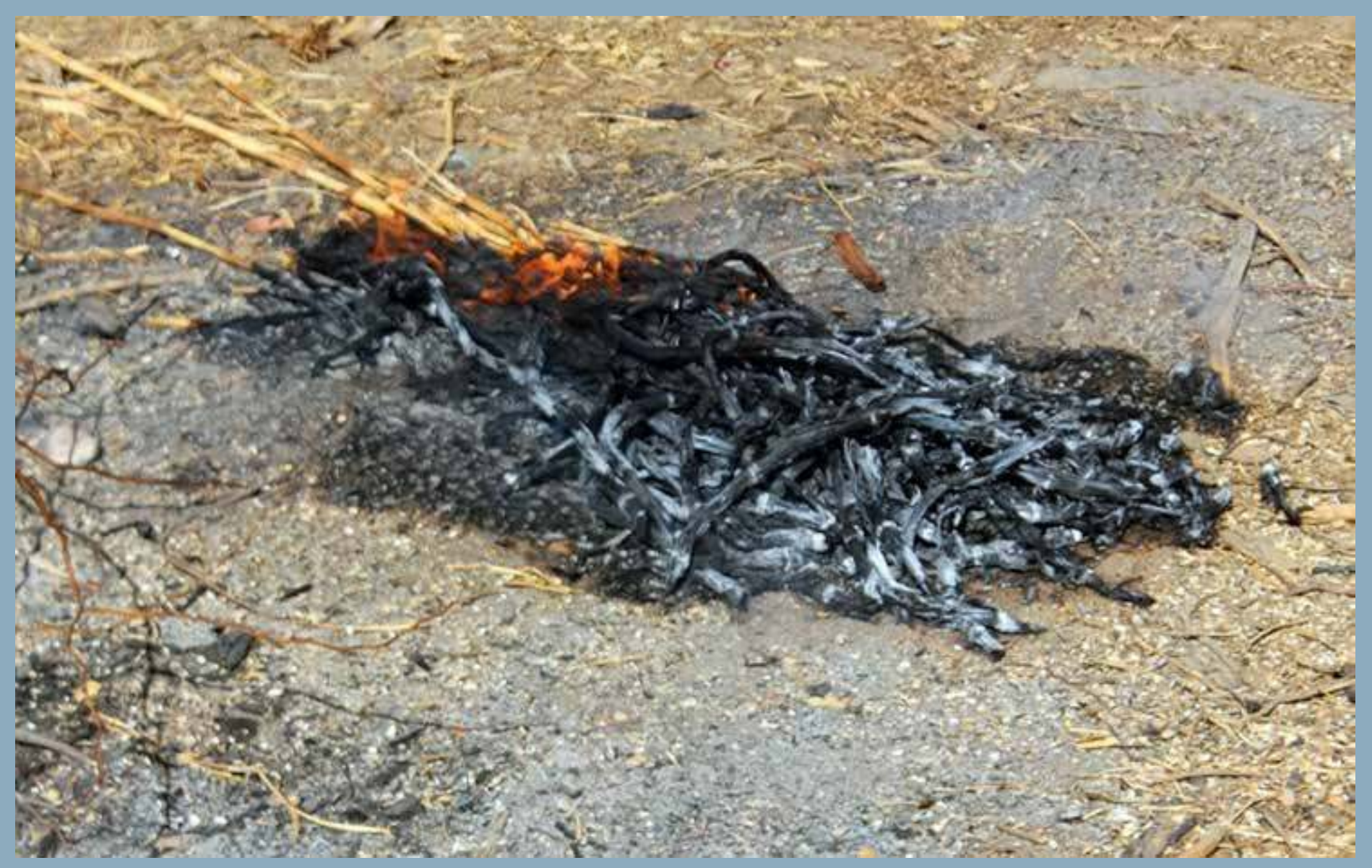




\section{La conservation du mil par la potasse}

\section{Vers une épistémologie dogon des matières}

Pour les communautés rurales dogons du Mali en Afrique de l'Ouest, la conservation des récoltes et des aliments cuits constitue un véritable défi quotidien. Le pays dogon est caractérisé par un climat de type sahélien marqué par de longues périodes de sécheresse et de fortes pluies, qui tombent de manière irrégulière spatialement et temporellement. De plus, dans certaines zones, les sols s'érodent et s'appauvrissent continuellement, rendant ainsi la culture du mil (pennisetum glaucum) de plus en plus difficile. Dans de telles conditions, les récoltes inégales et souvent faibles ne suffisent pas à nourrir les grandes familles (Douny 2014). Par ailleurs, avant et après les récoltes, les nuisibles, tels que les insectes et les petits rongeurs, mettent en péril la conservation du mil, laquelle reste essentielle, aujourd'hui encore, à la survie des Dogons du village de Kani Kombolé dans la région linguistique Tengu Kan. La difficulté pour beaucoup de familles d'accéder financièrement à des pesticides, mais également leur méfiance envers ces produits chimiques qui sont réputés nuire à la santé et altérer le goût du mil, fait que les Dogons ont recours à des techniques de conservation dites traditionnelles; l'usage de la cendre et de la potasse qui sont produites localement. Ces matières leur permettent ainsi de surmonter à court et à moyen terme, les nombreux problèmes de conservation de cette céréale vivrière et de certaines de ses préparations culinaires, mais aussi de les enrichir.

Comme le souligne Dumestre (1996: 689): «Le Mali est un pays où la faim et plus encore la peur de la faim sont encore des réalités présentes et pesantes. » Dans un tel contexte, conserver permet d'anticiper les mauvaises récoltes et le manque de nourriture, d'assurer les récoltes futures en préservant des semis soigneusement sélectionnés et en garantissant ainsi une certaine disponibilité quotidienne de nourriture (Sigaut 1978, Multon \& Sigaut 1982). Les techniques traditionnelles de conservation du mil les plus efficaces, formant un héritage de connaissances techniques, impliquent l'usage de la cendre et celui de la potasse, une matière résultant de la transformation de la cendre. La cendre de tiges de mil permet de conserver les épis, les graines et les semis de mil dans les greniers tandis que la potasse joue un rôle de conservateur lorsqu'elle est ajoutée aux 
La potasse de mil, est produite à partir de la percolation lente des cendres de la tige de mil versées dans la poterie perforée du haut et de la cuisson du filtrage récolté dans la poterie du bas.

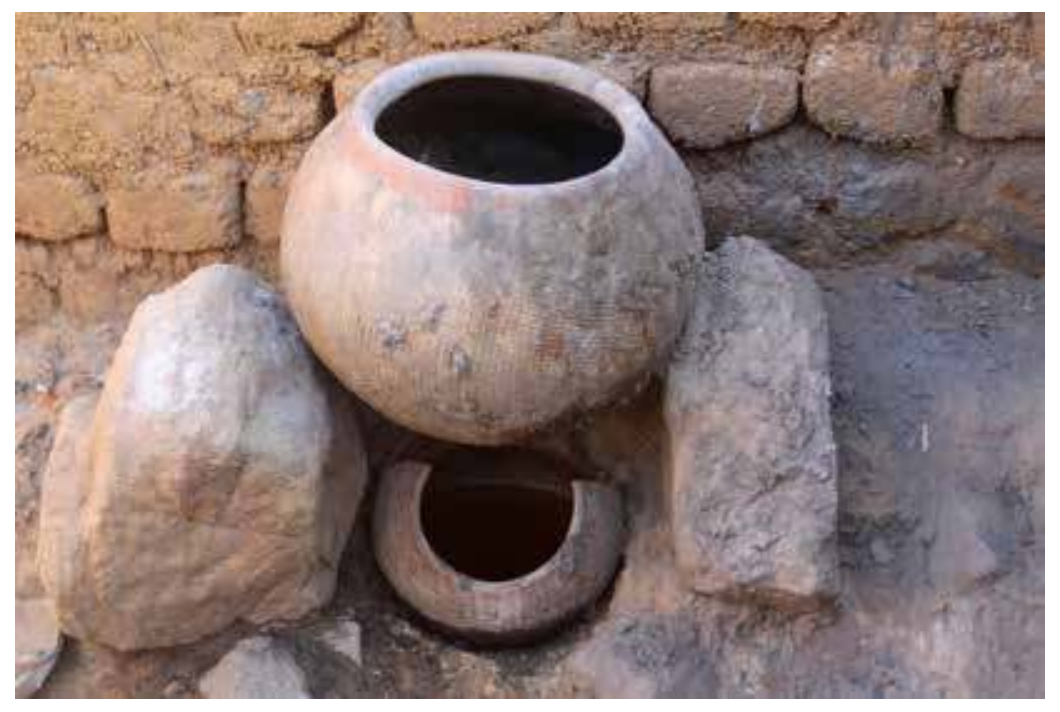

aliments cuits consommés quotidiennement telle que le ein nian, un repas préparé à base de mil écrasé sur une meule. Le terme «potasse » qui se dit ein en dialecte tengu kan, réfère généralement à toutes les matières alcalines ou amères (kakara ko) et plus précisément à la matière résultant de la transformation des cendres et qui est pourvue d'une «force» (panga na) ou substance active. La potasse, un sel végétal alcalin, est produite par un procédé de lixiviation des cendres de bois ou dans le cas présent de tiges de mil (dogné). En plus de conserver, les cendres et la potasse soignent, neutralisent les poisons et la sorcellerie (Tinta 2003), assaisonnent la nourriture et aussi fertilisent la terre. De plus, elles jouent un rôle important dans la transformation des matières brutes telles que la cuisson des cocons de soie indigène, la préparation du bain d'indigo servant à teindre les textiles ou la fermentation de l'adobe ${ }^{1}$ utilisé dans la construction des maisons et des greniers. Ainsi, les bénéfices de ces matières totalisantes et génératives produites par les vieilles femmes dogons sont multiples tant au niveau de la vie quotidienne que dans les pratiques rituelles.

À travers une ethnographie des techniques de conservation du mil, nous nous concentrerons sur les chaînes opératoires de la transformation et de la conservation du mil par les cendres et la potasse afin de mettre en évidence deux choses: en premier lieu, les séquences de production de la cendre et de la potasse, mais aussi, en second lieu, les logiques pratiques et symboliques à l'œuvre dans la conservation du mil. Celles-ci interviennent à deux moments clés: d'une part, par la cendre et au niveau de l'engrangement des épis et des grains dans les greniers en terre et, d'autre part, par la potasse et lors de la préparation du plat à base de mil écrasé. La description de ces chaînes opératoires donne à voir tout ce qui se joue lorsque les femmes et les hommes de ce village rendent conservables les récoltes et leur produit alimentaire. Nous porterons une attention particulière aux aspects liés à la ritualisation des techniques et aux formes de significations implicites (Lemonnier 2012), dont la magie, qui font partie intégrante de la définition et des pratiques de conservation dogons du mil. Dans le cadre d'une épistémologie indigène des matières, nous nous intéressons à l'efficacité matérielle de la cendre et de la potasse telle qu'elle est envisagée par les Dogons, afin de comprendre la logique d'action qui sous-tend les techniques traditionnelles de conservation. Deux grandes idées s'en dégagent. Premièrement, la notion d'efficacité matérielle repose sur les connaissances qu'ont les femmes et les hommes des propriétés matérielles de la cendre et de la potasse. Ils les définissent comme une force intrinsèque (panga na), une force qui est présente dans les aliments et les 
transcendent indépendamment de leur force physiologique (Zahan 1995: 114). Deuxièmement, la notion d'efficacité matérielle se définit au travers de l'interprétation indigène des mécanismes de transformation des cendres. Ceux-ci impliquent une dynamique relationnelle des matériaux établie entre les différentes parties des plants de mil telles que leurs épis, leurs grains et leurs tiges, qui sont toutes transformables, transformées et utilisées. Finalement, nous posons la question de ce que ce mode de conservation fait au mil et à ceux qui le consomment. La réponse à cette question nous invite à prêter attention aux aspects sociaux du système technique de conservation dogon, aux croyances et aux logiques qui sont à l'œuvre. Nous soulignerons certains aspects de la temporalité du mil, une céréale qui, grâce à la potasse, se bonifie à court terme lorsque préparée et qui symboliquement ne diminue ni dans le grenier ni dans l'assiette.

\section{En ligne}

Retrouver l'article complet sur journals.openedition.org/tc: Techniques\&Culture 69 «Le temps des aliments».

\section{Notes}

1. Mélange d'argile et de matières organiques telles que la paille du fonio.

\section{Iconographie}

Image d'ouverture. La cendre de tige de mil est utilisée comme moyen de conservation des épis, des grains et aussi des semis de mil. Crédits des images: (C) Laurence Douny.

\section{I'auteure}

Laurence Douny, anthropologue, elle mène depuis 2001 des recherches approfondies sur l'écologie du paysage sahélien et la culture matérielle ouest-africaine. Ses travaux portent sur l'anthropologie des techniques et des matériaux qu'elle a publiés dans plusieurs articles et un livre intitulé Living in a Landscape of Scarcity. Materiality and Cosmology in West Africa (Left Coast Press / Routledge, 2014). La potasse est un thème commun qui a émergé de ses recherches sur l'alimentation, la soie indigène et la teinture à l'indigo. Elle est actuellement collaboratrice scientifique à l'Université libre de Belgique et chercheur honoraire à l'University College de Londres.

\section{Références}

Douny, L. 2014. Living in a Landscape of Scarcity. Materiality and Cosmology in West Africa. London: Routledge.

Dumestre, G. 1996 «De l'alimentation au Mali», Cahiers d'études africaines 36 (144): 689-702.

Lemonnier, P. 2012 Mundane Objects: Materiality and Nonverbal Communication. Walnut Creek: Left Coast Press.

Multon, J.-L. \& F. Sigaut 1982 «Historique et prospective des technologies de stockage et de conservation des grains et graines », Industries alimentaires et agricoles 12: 1157-1171.
Sigaut, F. 1978 Les réserves de grains à long terme. Techniques de conservation et fonctions sociales dans l'histoire. Paris: Éditions de la MSH.

Tinta, S. 2003 «Les conceptions de la transmission, de la contagion et de la prévention de la maladie en milieu dogon (Mali)» in D. Bonnet, Y. Jaffré dir. Les maladies de passage. La construction sociale des notions de transmission. Paris: Éditions. Karthala.

Zahan, D. 1995 Le feu en Afrique et thèmes annexes. Variations autour de l'œuvre de H.A. Junod. Paris: L'Harmattan.

\section{Pour citer l'article}

Douny, L. 2018 «La conservation du mil par la potasse. Vers une épistémologie dogon des matières», Techniques\& Culture 69 «Le temps des aliments», p. 34-37. 